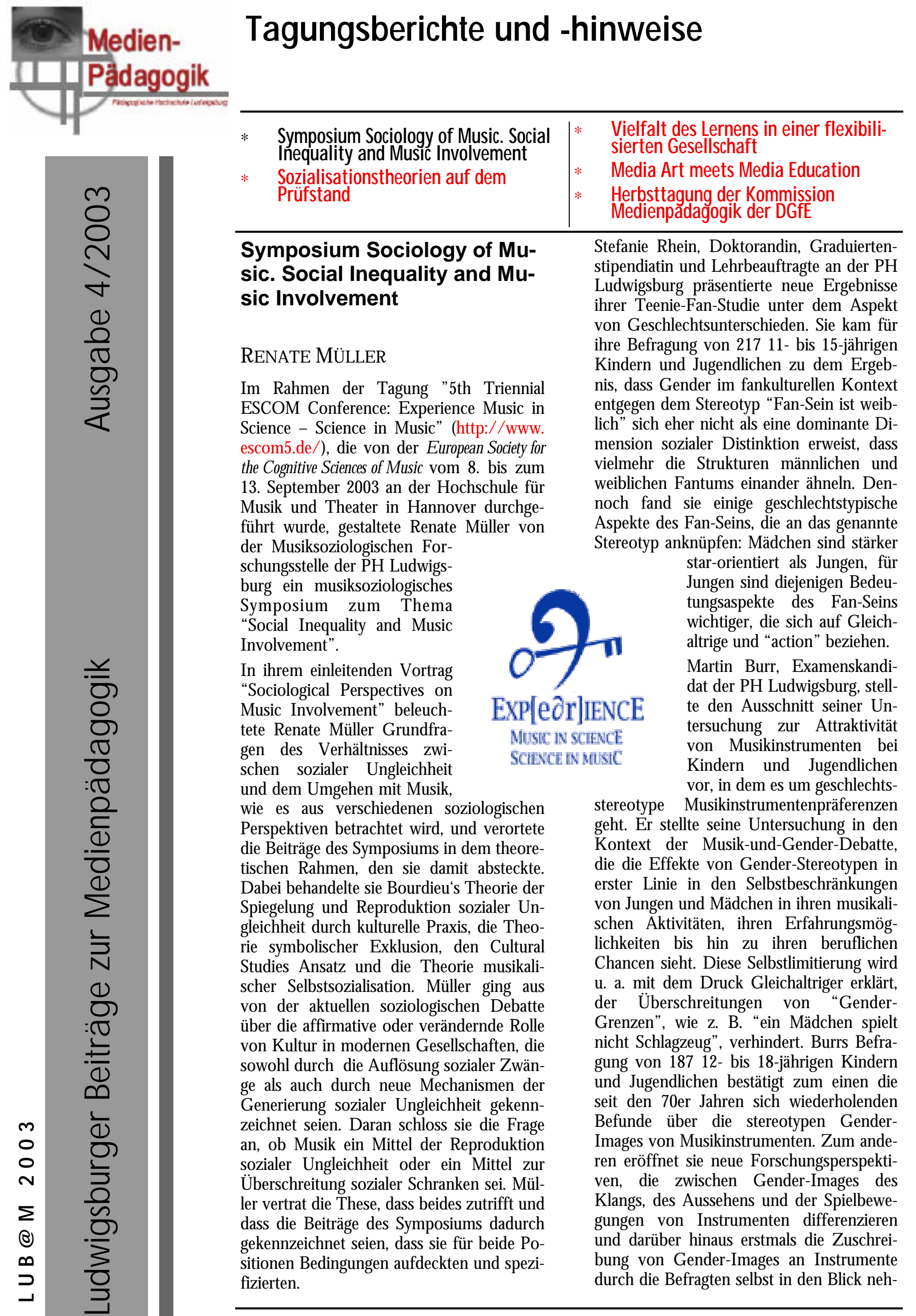


Hans Neuhoff, Westfälische WilhelmsUniversität Münster, war leider erkrankt, so dass er seine Forschungen über Konzertbesuch und soziale Ungleichheit nicht vorlegen konnte. Sein Vergleich zweier Publikumsuntersuchungen (1999 und 1979) ergab u. a., dass die Ungleichheitskategorie Alter nach wie vor am stärksten den Konzertbesuch beeinflusst. D arüber hinaus ergab Neuhoffs Studie über die zwischen den beiden Untersuchungen liegenden 20 Jahre hinweg eine deutlich Zunahme weiblicher Konzertaktivitäten.

Andreas Gebesmair, APART-Stipendiat der Österreichischen Akademie der Wissenschaften, Wien, referierte Ergebnisse seiner Studie zum Zusammenhang zwischen der Ausdifferenzierung des Musikgeschmacks und seiner Rolle als kulturelles Kapital bei der Reproduktion sozialer Ungleichheit. Er knüpft an die Reproduktionsthese Bourdieus an, die $u$. a. beinhaltet, dass ein "elitärer" Geschmack (symbolische Exklusion) Zutritt $\mathrm{zu}$ hohen gesellschaftlichen Positionen verschafft, von denen diejenigen ausgeschlossen sind, die einen "breiteren" Geschmack haben (soziale Exklusion). D emgegenüber vertritt Gebesmair im Anschluss an amerikanische Untersuchungen und Modifikationen der Theorie symbolischer und sozialer Exklusion die These, dass in modernen Gesellschaften gerade die Überschreitung von $\mathrm{G}$ eschmacksgrenzen einen neuen Mechanismus der Reproduktion sozialer Ungleichheit darstellt. Anhand einer Analyse von repräsentativen Daten aus dem ALLBUS 1998 mit ca. 2000 Befragten zeigt G ebesmair auf, dass Bildungsmobilität eng verknüpft ist mit einem breiteren (toleranten) musikalischen G eschmack, der $\mathrm{G}$ renzen zwischen Pop und Klassik transzendiert.

Die Vorträge bzw. Abstracts der gesamten ESCOM-Konferenz sind als CD-RO M erschienen:

Reinhard Kopiez, Andreas C. Lehmann, Irving Wolther and Christian Wolf (Eds.) (2003): Proceedings of the 5th Triennial Conference of the European Society for the Cognitive Sciences of Music (ESCO M5), Hanover University of Music and D rama, 8 - 13 September 2003. (ISBN: 3931852-67-9).

Burr, Martin (2003): Musical Instrument Preferences: $G$ ender-Images and $G$ ender Differences. In: Kopiez et al. 2003, 61 - 64.

Gebesmair, Andreas (2003): Musical Taste and Cultural Capital. In: Kopiez et al. 2003, 65- 68.

Müller, Renate (2003): Sociological Perspectives on Music Involvement. In: Kopiez et al. 2003, 52.

Neuhoff, Hans (2003): Concert Attendance and Social Inequality. In: Kopiez et al. 2003, 56.

Rhein, Stefanie (2003): Gender Differences in Teenage Fandom. A Survey on Musical Interaction in Fan Cultural Contexts. In: Kopiez et al. 2003, 56 - 61.

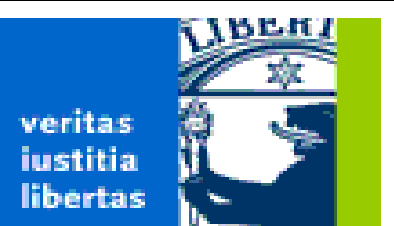

\section{Sozialisationstheorien auf dem Prüfstand}

Tagung der Sektion Jugendsoziologie der DGS vom 22.2.-24.2.2003 an der Freien

Universität in Berlin

\section{Organisation:}

DAGMAR HOFFMANN \& HANS MERKENS

Fachbereich Erziehungswissenschaft und Psychologie, Arbeitsbereich Empirische Erziehungswissenschaft, 14195 Berlin

\section{Verlauf der Tagung}

Die Sektion Jugendsoziologie (www. jugendsoziologie.de) der D eutschen G esellschaft für Soziologie (DGS, www.soziologie.de) hatte sich bei ihrer diesjährigen Tagung "Sozialisationstheorien auf dem Prüfstand" vorgenommen, darüber zu diskutieren, inwieweit verschiedene sozialisationstheoretische Ansätze zur Erklärung aktueller jugendtypischer Phänomene bzw. Verhaltensweisen zu Beginn des 21. Jahrhunderts (noch) brauchbar sind. Aus Sicht vieler Kollegen und Kolleginnen wurde damit eine längst überfällige D ebatte initiiert. So unterliegen die Bedingungen des Aufwachsens in den Gesellschaften westlicher D emokratien einem kontinuierlichen Wandel, der auch Veränderungen für Sozialisationsprozesse erwarten lässt. Ausgemacht werden Individualisierungs-, Diversifizierungs- und Pluralisierungsprozesse, sprich: strukturelle und kulturelle Differenzierungen und Neuorientierungen, die auch die Lebenswelt und Lebensbedingungen von Jugendlichen betreffen. Betrachtet man aktuelle Untersuchungen zur Jugendsozialisation, so stellt man fest, dass auf die gesellschaftlichen Veränderungen und damit auch individuell ausdifferenzierten Bedingungen des Aufwachsens nur bedingt eingegangen wird. Das Repertoire an Theorien zur Sozialisation von Jugendlichen hat sich in den letzten Jahrzehnten nur unwesentlich verändert. In Untersuchungen wird meist auf die klassischen Sozialisationstheorien verwiesen, die nur begrenzt weiterentwickelt und modifiziert wurden. Nach wie vor werden in der Sozialisationsforschung den traditionellen Instanzen der Sozialisation wie Familie, Schule und Peers große Bedeutung für die Entwicklung Jugendlicher beigemessen, wobei in jüngster Zeit zunehmend auch die Rolle der Medien diskutiert wird. Eine Modifikation gängiger Theorien könnte aber mitunter notwendig geworden sein, da sich die gesellschaftlichen Bedingungen des 
Aufwachsens so sehr verändert und ausdifferenziert haben, dass die klassischen Theorien zur Erklärung aktueller Phänomene und Problemlagen nur begrenzt geeignet sind. Die Tagung "Sozialisationstheorien auf dem Prüfstand" wurde von Dagmar Hofmann und Hans Merkens organisiert und fand im Senatssitzungssaal der Freien Universität statt. Unter den über 90 Teilnehmerinnen und Teilnehmern befanden sich auch solche aus Österreich, den Niederlanden, Schottland und der Schweiz. Da insgesamt 30 Vorträge präsentiert wurden, kann hier nicht auf alle einzelnen Beiträge eingegangen werden.

In den Eröffnungsvorträgen bilanzierten Matthias Grundmann, Hans Merkens, Andy Furlong und Matthias Junge aus unterschiedlichen Perspektiven die Implementierung soziologischer Sozialisationstheorien in die gegenwärtige Jugendforschung. Dieter Geulen, Hermann Veith und Dieter Kirchhöfer setzten sich in ihren Beiträgen mit der "Relevanz klassischer Sozialisationstheorien für die Bearbeitung aktueller Forschungsfragen" auseinander.

In drei parallel stattfindenden Arbeitsgruppen wurde über Forschungszugänge zu ausgewählten Sozialisationskontexten referiert und diskutiert. In der ersten Arbeitsgruppe fokussierten Klaus Boehnke, Christine Schmid sowie Heinz Reinders die politische Sozialisation im Jugendalter. In der zweiten Arbeitsgruppe wurde der gegenwärtige Forschungsstand zur Geschlechterrollensozialisation von Ursula Nissen, Hans-Peter Kuhn, Karin Flaake sowie Ulrike Popp bilanziert. Die dritte Arbeitsgruppe verhandelte aktuelle Forschungsparadigmen zur Mediensozialisation im Jugendalter. Zunächst berichtete Udo Göttlich aus verschiedenen Studien, an denen er be teiligt war, über die besonderen Motive der Rezeption von den bei Jugendlichen äußerst beliebten Genres: D aily Soaps, D aily Talks und "Big Brother". Lothar Mikos wies auf das Theoriedefizit in der Mediensozialisationsforschung hin und reflektierte kritisch die Konzeptionen von Medienkompetenz und Selbstsozialisation. Heinz Moser (Universität Zürich), würdigte in der Sektion "Mediensozialisation" den Cultural Studies Approach für das Erfassen medialer Sozialisationsprozesse. Abschließend evaluierte Bernd Schorb seine über einen langen Zeitraum vertretenen Konzeptionen von (Massen-)Mediensozialisation. Zusammen mit Helga Theunert sprach er sich für eine subjekt-handlungsorientierte Perspektive in der Mediensozialisationsforschung aus. Anhand einiger Teilergebnisse einer Studie zur Rezeption politischer Informationen verdeutlichte er die Notwendigkeit dieser theoretischen Herangehensweise.

Abgeschlossen wurde die dreitägige Tagung mit Beiträgen von Hannelore Faulstich-Wieland, Vera King, Albert Scherr, Renate Müller, Patrick Glogner und Stefanie Rhein sowie von Henk
Vinken zum Thema "Neue Konzepte der Sozialisation von Jugendlichen und zur Debatte um die Selbstsozialisation".

\section{Resumée}

Die Tagung erbrachte somit eine aktuelle Bestandsaufnahme zu den gegenwärtigen Verhandlungen in den Sozialwissenschaften über den Themenbereich "Jugend und Sozialisation". D abei sind sehr unterschiedliche Sichtweisen aus verschiedenen Disziplinen berücksichtigt worden. Zudem haben die ausländischen Gäste über den Stand nationaler Diskurse berichten und diesen in die allgemeine Diskussion, wie sie sich bei den Jugendsoziologen darstellt, integrieren können. Insgesamt wurde die Notwendigkeit der Überprüfung bestehender Sozialisationstheorien bestätigt.

Die Ergebnisse können wie folgt zusammengefasst werden: Nach einer ausführlichen „Evaluation' klassischer Sozialisationstheorien im Hinblick auf die empirische Brauchbarkeit für aktuelle jugendtypische Phänomene und Problemlagen, werden mehrheitlich interaktionistische Sozialisationstheorien für zeitgemäß und aussichtsreich für aktuelle Forschungsparadigmen befunden - insbesondere im Hinblick auf Fragestellungen in den Bereichen Geschlechterrollen- und Mediensozialisation. Prinzipiell scheint es aber unabdingbar, Abgrenzungen zu subjekt-handlungsorienierten und sozialkonstruktivistischen Ansätzen vorzunehmen und/ oder Kongruenzen aufzuzeigen. Die D ebatte um Selbst- bzw. Fremdsozialisation reagiert auf die Nichtberücksichtigung von Individialisierungs- und Modernisierungsprozessen in bisherigen theoretischen Konzepten von Sozialisation. Das eigentliche Theoriedefizit hebt sie jedoch nicht auf und eine ,Reformierung' der Sozialisationstheorien treibt sie nur bedingt voran. Wichtig scheint, dass eine soziologisch orientierte Sozialisationstheorie, auch eine Art Instanzen- bzw. Institutionenforschung erlaubt. D abei gilt es die Akteursperspektive mit einzubeziehen und explizit gesellschaftliche Gegenwartsanalysen $\mathrm{zu}$ berücksichtigen. Es sollte extensiv über eine effektive Verknüpfung von makrosozialen Strukturen und mikrosozialen Entwicklungsprozessen nachgedacht werden und darüber, wie man diese in Sozialisationstheorien implementieren kann.

In den Arbeitsgruppen wurde die heterogene Verknüpfung von Theorie und empirischer Forschung deutlich. Die Arbeitsgruppe "Mediensozialisation" fragte eingehend nach der Existenz der Sozialisationsperspektive in der Massenkommunikationsforschung bzw. Jugendmedienforschung. D as Fazit der D iskussionen war, dass eine solche nach wie vor vernachlässigt wird und längst nicht selbstverständlich ist. Unstrittig ist, dass Medien eine Instanz der Sozialisation sind und zwar nicht nur für Jugendliche. Es bestehen aus handlungsorientierter Sicht diffuse Interde- 
pendenzen zwischen Gesellschaft, Medien und Individuum, die expliziten und impliziten Wirkmechanismen bzw. Aneignungsprozesse sind allerdings in bezug auf Sozialisationsverläufe weitgehend unklar. Plädiert wurde für die Implementierung des Cultural Studies Approachs, der ein erprobtes Konzept qualitativer Forschung sei, das Objektivität vermeidet (aus einer Kultur heraus, soziale Phänomene (Mediennutzung) erklärt), das sowohl den Anbieter wie den Rezipienten - anders formuliert den Sozialisanden wie auch den Sozialisationsagenten - über das Encoding/ D ecoding-Modell mitdenkt. Allerdings sind die Schwierigkeiten der methodischen Umsetzbarkeit noch existent, will man diesen theoretischen Ansatz in Gänze und nicht partikular anwenden. Außerdem wurde in der Sektion der für Sozialisationsprozesse sehr wichtige Begriff der Kompetenz diskutiert. Der Begriff der Medienkompetenz ist - operationalisiert nach Baacke und Groeben - weitgehend abgekoppelt von sozialisationstheorischen Überlegungen. Es besteht das Diktat der Ausbildung einer Kunde über Medien, des Umgangs mit Medien und der Medienkritik. Die Frage, die sich in dem Zusammenhang stellt, ist die nach der ,gleichberechtigten' Position von Medien im Verbund mit anderen Instanzen der Sozialisation. Schließlich ist auch nicht von einer "Familienkompetenz" oder "Schulkompetenz" die Rede.

Zusammenfassend lässt sich berichten, dass die Tagung "Sozialisationstheorien auf dem Prüfstand" sowohl von den Referenten und Referentinnen als auch den Teilnehmern und Teilnehmerinnen als ausgesprochen gut organisiert bewertet worden ist. Die Disziplinienung der Redner und Rednerinnen im Hinblick auf die Redezeit fand lobende Erwähnung, da dadurch stets ausreichend Zeit zur Diskussion blieb, was als sehr angenehm empfunden wurde und diese Veranstaltung von daher nicht den Charakter eines "Vortragsmarathons" hatte - wie leider inzwischen sonst oft üblich. Begrüßt wurde das Zusammenbringen verschiedener $G$ enerationen von Wissenschaftlern und Disziplinen. So diskutierten renommierte Professoren mit jungen Nachwuchswissenschaftlem und -wissenschaftlerinnen konstruktiv über Modifikationsmöglichkeiten klassischer Sozialisationstheorien und über die Chancen neuer sozialisationstheoretischer Konzepte. Somit wurde eine wichtige Debatte innerhalb der Jugendsoziologie über die Überwindung der Diskrepanzen zwischen Sozialisationstheorie und Forschungspraxis mit Unterstützung anderer Disziplinen inklusive der "BindestrichSoziologien" um bedeutsame Aspekte angereichert und auch weiterentwickelt.

Dagmar Hoffmann und Hans Merkens (gekürzt von Renate Müller, erscheint ungekürzt in einem der nächsten Hefte in: Soziologie. Forum der Deutschen Gesellschaft für Soziologie)
Die Tagungsbeiträge werden im Frühjahr 2004 in der der Reihe Jugendforschung im JuventaVerlag veröffentlicht: Hoffmann, D agmar/ Merkens, Hans (Hrsg.): Sozialisationstheorien auf dem Prüfstand. Weinheim und München: Juventa (im D ruck).

Der Reihe Jugendforschung im Juventa-Verlag veröffentlicht: Hoffmann, Dagmar / Merkens, Hans (Hrsg.): Sozialisationstheorien auf dem Prüfstand. Weinheim und München: Juventa (im D ruck). 


\section{Vielfalt des Lernens in einer flexibilisierten Gesellschaft}

Frühjahrstagung der Sektion „Jugendsoziologie" der DGS zusammen mit dem Deutschen Jugendinstitut in München am 22. und 23. März 2004

\section{Renate MÜLleR}

\section{Call for Papers}

Lemprozesse sind heute nichts zuletzt infolge der Mediatisierung und Flexibilisierung sozialer Bezüge durch einen hohen Grad der "Informalisierung" gekennzeichnet. Wie sich unter diesen Bedingungen die Aneignung von Wissen und Kompetenzen vollzieht, ist bisher nur wenig bekannt. Dieses Forschungsdefizit ist auch für Jugendforschung von Bedeutung, denn gerade der Jugendalltag, unabhängig davon, ob dieser Lebensabschnitt als eigenständige Lebensphase oder als Vorbereitung auf das Erwachsenendasein und Berufsleben erachtet wird, kann als höchst lernintensiv bezeichnet werden. Die z. T. technisch induzierten veränderten Lernanforderungen besitzen bislang noch nicht vollständig prognostizierbare Herausforderungen. Es wird zwar häufig versucht, passende Bezeichnungen für einen Wandel der Lernkultur zu finden (z. B. selbstgesteuertes, informelles oder lebenslanges Lernen), doch bleibt wie so häufig die Verbindung von wortgewaltiger Diagnose, theoretisch gehaltvoller Analyse und praktisch-pädagogischer Umsetzung aus.

Mit der Tagung soll auf diesen Umstand reagiert werden. In den Beiträgen sollten die folgenden Punkte fokussiert werden:

a) Die Dynamisierung der Gesellschaft als Auslöser der Informalisierung. Hierbei geht es um die soziologische Deutung der Gegenwartsgesellschaft, vor deren Hintergrund sich sowohl die Jugend, als auch das Lernen verändern.

b) Lernwelten in und neben der Schule. Zu fragen ist, inwieweit die Schule als Ort institutionalisierter Lernprozesse selbst einem Wandel unterworfen ist und welche neuen Lernfelder sich im Jugendalltag ausdifferenzieren (z.B. Technik, Nebenjob, Sport etc.).

c) Grenzen und Probleme des Lernens in der flexibilisierten G esellschaft. Hier spielen u.a. Beobachtungen pädagogischer Provenienz eine Rolle, die möglicher Weise die Überforderung von Jugendlichen oder das Aufscheinen neuer Lem-Ungleichheiten diagnostizieren.
Referatsangebote sollten spätestens bis zum 10. Januar 2004 mit Betreff "Informalisierung" bei Claus J. Tully Deutsches Jugendinstitut, Nockherstr. 2, 81541 München eingehen.

Email: tully@ dji.de

Tel.: 089/ 62306(0) -190 


\section{Künstlerische Impulse}

für die Medienpädagogik

media art meets

media education

\section{Media Art meets Media Education}

\section{HORST NIESYTO}

Künstlerische Impulse für die Medienpädagogik, Brücken zwischen Medienpädagogik und $\mathrm{Me}$ dienkunst bauen - dies sind wichtige Intentionen, die sich mit dem 20. G MK-Forum Kommunikationskultur verbinden. D as Forum findet als bundesweite Veranstaltung mit internationaler Beteiligung vom 21-23. November 2003 an der Hochschule für Film und Fernsehen Potsdam Babelsberg statt. In mehreren Plenumsvorträgen, 10 Workshops, einer Podiumsdiskussion, einem "Offenen Forum" und einer Ausstellung werden konzeptionelle Überlegungen und viele Praxisbeispiele zum Thema des Forums vorgestellt.

Idee und Rahmenkonzept wurden von Prof. Dr. Horst Niesyto (PH Ludwigsburg / GMK-Vorstand) entwickelt und in einer Vorbereitungsgruppe konkretisiert. Die Veranstaltung wird von der GMK in Kooperation mit dem Zentrum für Kulturforschung Bonn (Programmträger des BLK-Programms "Kulturelle Bildung im Medienzeitalter") durchgeführt.

Programm-Infos und Anmeldung:

http:/ / www.gmk-net.de/

Rahmenkonzept:

http:/ / www.gmk-net.de/ forum.htm

\section{DGfE}

\section{Herbsttagung der Kommission Medienpädagogik der Deutschen Gesellschaft für Erziehungswis- senschaft}

\section{HoRST NIESYTO}

Am 27. und 28. November 2003 findet an der O tto-von-G uericke-Universität Magdeburg (Institut für Erziehungswissenschaft) die diesjährige Herbsttagung der Kommission Medienpädagogik statt. Im Mittelpunkt stehen folgende Themen: 1. Kerncurriculum Erziehungswissenschaft und Kerncurriculum Medienpädagogik, 2. Forschungsmethoden anhand aktueller Projekte. Zum Punkt 2 werden seitens der PH Ludwigsburg Prof. Dr. Horst Niesyto und Dipl.-Päd. Peter Holzwarth einen Vortrag halten: "Qualitative Forschung auf der Basis von Eigenproduktionen mit Medien - Erfahrungswerte aus dem aktuellen EU-Forschungsprojekt CHICAM“. Genaues Programm incl. DownloadMöglichkeit von Abstracts und Manuskripten: siehe unter

http:/ / www.uni-kassel.de/ fb1/ mediafb1/ dgfemedien/ Seiten/ infos.shtml 